\title{
Efficient Evaluation of Multichannel SAR Data Recombination Filters
}

\author{
Moritz Kiemer and Helko Breit
}

\begin{abstract}
Synthetic aperture radar (SAR) is a well-established technique for observing the Earth on a global scale. As applications become more demanding, it is desirable to overcome the limitations imposed by the SAR principle, one of which is the tradeoff between the swath width and the instantaneous azimuth bandwidth, determining the resolution. Recombination of multiple channels with displaced phase centers has been proposed as a convenient way to create high resolution wide-swath images. We analyze various approximations made in the channel transfer functions and their impact on the reconstruction result using examples inspired by current imaging modes of the TerraSAR$X$ and TanDEM-X missions. In order to do so, we introduce an efficient method to assess the quality of reconstruction filters for an arbitrary number of channels without the need of full time-domain simulations.
\end{abstract}

Index Terms-Radar signal processing, signal reconstruction, spaceborne radar, synthetic aperture radar (SAR).

\section{INTRODUCTION}

$\mathbf{I}$ N CONVENTIONAL synthetic aperture radar (SAR) imaging, the unambiguous azimuth frequency bandwidth is determined by the pulse repetition frequency (PRF) of a SAR sensor. Since the echo of a pulse coming from far range should not superimpose the near range echo of a subsequent pulse, the maximal swath width is inversely proportional to the PRF. This results in the well-known conflict between azimuth resolution and swath-width of classical SAR imaging modes [1, 4.5.3]. Several techniques have been developed to overcome this limitation.

Currently, wide swath modes are realized via beam steering, namely, by scanning several subswathes alternatingly in flight direction. These modes are known as ScanSAR and Terrain Observation by Progressive Scans. The latter has been implemented experimentally on TerraSAR-X [2] and is the main operational mode on Sentinel-1 [3].

On future SAR systems, elevation beam forming could be used to resolve the range ambiguities within one echo window, while the blind spots corresponding to the transmit events are shifted in range by means of a varying PRF, as described in [4] and [5].

Manuscript received September 21, 2016; revised February 16, 2017 and May 10, 2017; accepted July 3, 2017. Date of publication August 25, 2017; date of current version October 25, 2017. (Corresponding author: Moritz Kiemer.)

M. Kiemer is with the Department of Photogrammetry and Remote Sensing, Technische Universität München, 80333 Munich, Germany (e-mail: moritz.kiemer@dlr.de).

H. Breit is with the Remote Sensing Technology Institute, German Aerospace Center, 82234 Weßling, Germany.

Color versions of one or more of the figures in this paper are available online at http://ieeexplore.iee.org.

Digital Object Identifier 10.1109/TGRS.2017.2724918
Brown [6] showed that a low-pass signal of bandwidth $B$ can be uniquely described by $m$ suitable channels, for each of which a sampling frequency of $B / m$ is sufficient. Following this mathematical principle, we can also increase the azimuth frequency by employing multiple receivers and using these different channels to reconstruct a higher sampled version.

This technique, known as multichannel azimuth processing, has been described in various papers [7], [8] using simulated data, and was applied to data acquired by the Dual Receive Antenna (DRA) mode of TerraSAR-X in [9].

Multichannel azimuth reconstruction can be applied to a variety of configurations more complex than the DRA mode. We will investigate the impact of system operating parameters and approximation errors in the channel transfer functions on the reconstruction results.

There are numerous variables involved in the overall performance of multichannel systems, such as the number of channels, the position and movement of the receivers relative to each other, different steering modes, and system parameters such as the antenna pattern and the PRF. Therefore, it is desirable to evaluate different processing scenarios without the need for computationally demanding time-domain simulations and the implementation of different processors.

This is achieved by simulating and reconstructing targets directly in the spectral domain, similar to the methodology proposed in [10]. The required patch size of the simulation is then reduced to the patch required for the final timedomain representation of the focused point target response. Analyzing the focused signals, we can assess the quality of the reconstruction filter, thereby determining limitations and performance drivers for multichannel SAR system configurations along with accuracy requirements for the reconstruction filters.

The analysis of the recombination filters is based on the assumption that the signal model presented in Section II-A appropriately describes the received signal, and thus measures the theoretical performance of a reconstruction scheme.

The outline of the remaining paper is as follows. Section II explains how the data is simulated, by first introducing the signal model (Section II-A), and then describing how the spectral representation is used to compute the aliased channels (Section II-B). The reconstruction theory is summarized in Section III-A, and the chosen reconstruction method in Section III-B, where a generic expression for the channel transfer functions is also established [see (24)]. In Section IV, several configurations of two-channel systems (along track separation in Section IV-A and cross track separation in Section IV-B) and their reconstruction options are analyzed. 
TABLE I

SAR SYMBOLS

\begin{tabular}{|c|c|}
\hline Symbol & Meaning \\
\hline \hline$t$ & slow time (azimuth) \\
\hline$f$ & Doppler frequency (azimuth) \\
\hline$\tau$ & fast time (range) \\
\hline$\nu$ & range frequency \\
\hline$\nu_{0}$ & radar frequency \\
\hline$c$ & speed of light \\
\hline $\mathrm{j}$ & imaginary constant \\
\hline
\end{tabular}

Special attention is directed to the spatial variation of the reconstruction filters.

\section{A. Signal Model}

\section{Simulation}

Throughout this paper, we will use the symbols defined in Table I.

Consider the echo of a single point scatterer, located at geometrical closest-approach coordinates $\left(\tau_{0, \text { geo }}, t_{0, \text { geo }}\right)$, and suppose we have $n_{\mathrm{CH}}$ independently recorded channels. For each channel $i \in\left\{0, \ldots, n_{\mathrm{CH}}-1\right\}$, there are channel parameters $A_{i}, \tau_{0, \text { fit }, i}$, and $t_{0, \text { fit }, i}$ such that the signal delay time is given by

$$
\tau_{\text {echo }}(t)=\sqrt{A_{i}\left(t-t_{0, \text { fit }, i}\right)^{2}+\tau_{0, \text { fit }, i}^{2}} .
$$

As suggested by the index "fit," and by choosing the dimensionless " $A$," these parameters are determined by performing a hyperbolic fit to the range delay function. They are purely mathematical and do not show physical meaning. However, they must accurately describe the signals properties, and thus account for effects like those of the platform motion and the troposphere. If necessary, this can be accomplished by updating the parameters along different subapertures, to better fit to the curved orbit (as shown in [10]).

Transforming the resulting time signals for each channel to the spectral domain and including terms $E(\nu, f)$ and $G(v)$ representing the 2-D antenna pattern and the transmitted range pulse, respectively, we arrive at the classical SAR signal model, given by

$$
\begin{gathered}
S_{i}(\nu, f)=E_{i}(\nu, f) \cdot G(v) \cdot \exp \left\{-2 \pi \mathrm{j} t_{0, \text { fit }, i} f\right\} \\
\exp \left\{-2 \pi \mathrm{j}\left(\nu+v_{0}\right) \tau_{0, \mathrm{fit}, i} \sqrt{1-\frac{f^{2}}{\left(v+\nu_{0}\right)^{2} A_{i}}}\right\} .
\end{gathered}
$$

However, the individual channels can also be considered to be filtered versions of a common (virtual) reference signal,

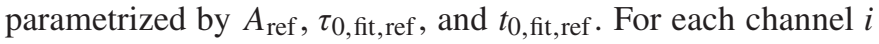
a time-invariant transfer function $H_{i}$, which acts upon the reference signal to yield the corresponding channel signal, can be defined by

$$
S_{i}(\nu, f)=H_{i}(\nu, f) \cdot S_{\text {ref }}(v, f) .
$$

Note that this is only possible if the support of $E_{i}(\nu, f)$ is a subset of the support of $E_{\text {ref }}(\nu, f)$.

After the signals are sampled with sampling frequency PRF upon receiving, their spectra are aliased and periodic, and can be expressed as a function defined on an arbitrary interval $I$ of length PRF by

$$
\mathcal{S}_{i}(v, f)=\sum_{z \in \mathbb{Z}} S_{i}(v, f+z \mathrm{PRF}), \quad f \in I .
$$

Of course the sum has only a finite number of nonzero summands due to the band limitation of $S_{i}$.

\section{B. Computation of the Simulated Spectrum}

The key point of channel recombination filters is that the considered signal bandwidth is wider than the sampling frequency of the recorded channels. For realistic results, the simulated bandwidth has to be chosen wide enough, such that the signal's energy beyond it can safely be ignored.

Note that throughout the following, " $B$ " will denote frequency intervals, not bandwidths. For convenience, we will extend the azimuth simulation band $B_{S}$ to a width of an integer multiple of the PRF. Writing $f_{S \text {, min }}$ for the minimal simulation frequency, and dividing the interval $B_{S}$ spanning the desired simulation bandwidth into $n_{S}$ azimuth subbands of width PRF, we define

$$
B_{S}=\bigcup_{j=0}^{n_{S}-1} B_{S, j}
$$

where the $j$ th simulation subband is given by

$$
\left.\left.B_{S, j}=\right] f_{S, \min }+j \cdot \mathrm{PRF}, f_{S, \min }+(j+1) \cdot \mathrm{PRF}\right] \text {. }
$$

When choosing the above, it should be kept in mind that the reconstructed bandwidth will consist of successive subbands chosen from the $B_{S, j}$.

We can now simplify the description of $\mathcal{S}_{i}$ in (4) by assuming that $S_{i}$ vanishes outside $B_{S}$ (thus keeping only a finite number of summands), and for the sake of clarity describing the resulting coherent sum as a function defined on the interval $B_{S, 0}$

$$
\mathcal{S}_{i}(v, f)=\sum_{j=0}^{n_{S}-1} S_{i}(\nu, f+j \mathrm{PRF}), \quad f \in B_{S, 0} .
$$

In order to properly deal with the arising aliasing, we will consider every function defined on the real numbers $\mathbb{R}$ as being "stitched together" by parts defined on $B_{S, 0}$. The interval $B_{S, 0}=\left[f_{S, \min }, f_{S, \min }+\mathrm{PRF}[\subset \mathbb{R}\right.$ induces a ring isomorphism $\mathcal{I}$ from complex valued functions $S$ defined on $\mathbb{R}$ to sequences of functions defined on $B_{S, 0}$

$$
\begin{aligned}
\mathcal{I} & : \mathcal{F}(\mathbb{R}, \mathbb{C}) \stackrel{\sim}{\longrightarrow} \mathcal{F}\left(B_{S, 0}, \mathbb{C}\right)^{\mathbb{Z}} \\
\quad & : S(x) \mapsto\left(\left.S_{z}(x-z \cdot \mathrm{PRF})\right|_{B_{S, 0}}\right)_{z \in \mathbb{Z}}
\end{aligned}
$$

where $S_{z}(x)$ is defined by

$$
S_{z}(x):=S(x+z \cdot \mathrm{PRF}) .
$$

Since in the following considerations, functions will have bounded support (the bandwidth of the considered signals is finite), we can restrict ourselves to a finite subset $J$ of $\mathbb{Z}$ and write the functions as vectors with $|J|$ elements. Aliasing and reconstruction are now represented as matrix operations with coefficients in $\mathcal{F}\left(B_{S, 0}, \mathbb{C}\right)$. 
We will use the index " $j$ " to refer to the respective simulation subband, and define

$$
S_{i, j}(\nu, f):=S_{i}(\nu, f+j \mathrm{PRF}) .
$$

$S_{\text {ref }, j}(\nu, f)$ and $H_{i, j}(\nu, f)$ are defined accordingly.

The number of azimuth samples for which (7) is computed should be chosen reasonably high to result in a wrapping free focused point target after reconstruction and focusing.

\section{RECONSTRUCTION}

\section{A. Theory}

According to [6], we may be able to recover a signal from its channel signals, if the original signal is band limited to the product of the number of channels and their common sampling frequency.

In a multichannel system, the exact recovery condition corresponds to a band limitation of $s_{\text {ref }}$ to $n_{\mathrm{CH}}$.PRF. In general, of course, the considered azimuth signal is not bandlimited in this manner. The degree to which this criterion is violated depends on the width of the azimuth antenna patterns, and the artifacts induced by this "residual aliasing" can be evaluated using the method under discussion, as $n_{\mathrm{CH}}<n_{S}$ is assumed throughout this paper.

For now, consider the general case where we want to recover the signal on a successive set of $n_{R}$ subbands chosen from $B_{S, j}$, corresponding to an ambiguous sampling frequency of $n_{R}$. PRF. As stated above, the theoretical limit for $n_{R}$ is $n_{\mathrm{CH}}$. Also choose a band offset $0 \leq n_{O} \leq n_{S}-n_{R}$, such that the spectral reconstruction domain is given by

$$
B_{R}=\bigcup_{k=0}^{n_{R}-1} B_{S, n_{O}+k} .
$$

Restricting the isomorphism induced by the lowest simulation band $B_{S, 0}$ to the reconstruction band $B_{R}$, we write the reconstructed signal spectrum $\mathcal{S}_{R}$ as

$$
\mathcal{S}_{R}(v, f)=\left(\begin{array}{c}
S_{0, R}\left(v, f-n_{O} \mathrm{PRF}\right) \\
\vdots \\
S_{n_{R}-1, R}\left(v, f-\left(n_{O}+n_{R}-1\right) \mathrm{PRF}\right)
\end{array}\right) .
$$

For any given set of recombination filters $P_{k, i} \in \mathcal{F}\left(B_{S, 0}, \mathbb{C}\right)$ $\left(0 \leq k<n_{R}\right.$ and $\left.0 \leq i<n_{C H}\right)$, we can define $n_{R}$ reconstructed parts of the spectrum as linear combinations of the aliased channels

$$
S_{k, R}(v, f):=\sum_{i=0}^{n_{\mathrm{CH}}-1} P_{k, i}(v, f) \cdot \mathcal{S}_{i}(v, f) .
$$

Using (3) and (4), we obtain a set of $n_{R}$ equations

$$
\begin{aligned}
S_{k, R}(v, f) & := \\
& =\sum_{i=0}^{n_{\mathrm{CH}}-1} P_{k, i}(v, f) \cdot\left(\sum_{j=0}^{n_{S}-1} H_{i, j}(v, f) S_{\mathrm{ref}, j}(v, f)\right) \\
& =\sum_{j=0}^{n_{S}-1} \sum_{i=0}^{n_{\mathrm{CH}}-1} P_{k, i}(\nu, f) H_{i, j}(v, f) S_{\mathrm{ref}, j}(v, f)
\end{aligned}
$$

all defined for $f \in B_{S, 0}$. Writing $\mathbb{P}$ and $\mathbb{H}$ for the matrices consisting of the $P_{k, i}$ and $H_{i, j}$, respectively, this can be written as the matrix equation

$$
\mathcal{S}_{R}(v, f)=\mathbb{P H}\left(\begin{array}{c}
S_{\mathrm{ref}, 0} \\
\vdots \\
S_{\mathrm{ref}, n_{S}-1}
\end{array}\right) .
$$

We will refer to the $n_{R} \times n_{S}$ matrix $\mathbb{P H}$ as "reconstruction scheme" $\mathbb{S}$ in the following considerations. It describes how multichannel sampling and reconstruction, represented by $\mathbb{H}$ and $\mathbb{P}$, respectively, act upon the postulated reference signal. The $(k, j)$ th entry of $\mathbb{S}$ describes the contribution of the signal defined on the $j$ th simulated subband to the $k$ th reconstructed one. In order to choose appropriate reconstruction filters $\mathbb{P}$, we will discuss what the reconstruction scheme should look like.

The ideal reconstructed spectrum-perfectly recovering the unambiguous signal within the desired reconstruction bandwidth—is denoted by $\mathcal{S}_{I}$ and defined within $B_{R}$ as

$$
\mathcal{S}_{I}(v, f)=\left.S_{\mathrm{ref}}\right|_{B_{R}}(v, f)=\left(\begin{array}{c}
S_{\mathrm{ref}, n_{O}}(v, f) \\
\vdots \\
S_{\mathrm{ref}, n_{O}+n_{R}-1}(v, f)
\end{array}\right) .
$$

Clearly, the condition for perfect reconstruction (i.e., $\mathcal{S}_{R}=\mathcal{S}_{I}$ ) is that the reconstruction scheme corresponds to a projection $\mathbb{S}_{P}$ of the original $n_{S}$ subbands onto the $n_{R}$ reconstructed ones. Since the subbands from $n_{O}$ to $n_{O}+n_{R}-1$ are reconstructed, this is the case if $\mathbb{S}_{P}$ consists of rows $n_{O}$ to $n_{O}+n_{R}-1$ of the $n_{S}$ by $n_{S}$ identity matrix

$$
\mathbb{S}_{P}=\left(\delta_{\left(k+n_{O}\right)=j}\right)_{\substack{0 \leq k<n_{R} \\ 0 \leq j<n_{S}}}
$$

(in case $n_{S}=n_{\mathrm{CH}}=n_{R}$ this means that $\mathbb{P}$ is the inverse of $\mathbb{H}$ ).

Note that the ideal spectrum $\mathcal{S}_{I}$ does not include any of the aliased signal components that would be present, had the reference signal been sampled with a frequency of $n_{R}$. PRF. A more "realistic" reconstruction should include ambiguities due to the aliasing with respect to the new signal sampling frequency $n_{R}$. PRF. It is represented by a matrix $\mathbb{S}_{A}$ where the diagonal entries of $\mathbb{S}_{P}$ recur at every $n_{R}$ th position [for a simple example refer to (32)]

$$
\mathbb{S}_{A}=\left(\delta_{\left(k+n_{O}\right) \equiv j\left(\bmod n_{R}\right)}\right)_{\substack{0 \leq k<n_{R} \\ 0 \leq j<n_{S}}}
$$

Using these matrices, we can express the total ambiguous signal component $S_{A}$ of the reconstructed signal by

$$
S_{A}(v, f)=\left(\mathbb{P H}-\mathbb{S}_{P}\right)\left(\begin{array}{c}
S_{\mathrm{ref}, 0} \\
\vdots \\
S_{\mathrm{ref}, n_{S}-1}
\end{array}\right)
$$

and the ambiguous signal component added by the reconstruction process itself $S_{A R}$ by

$$
S_{A R}(v, f)=\left(\mathbb{P H}-\mathbb{S}_{A}\right)\left(\begin{array}{c}
S_{\mathrm{ref}, 0} \\
\vdots \\
S_{\mathrm{ref}, n_{S}-1}
\end{array}\right) .
$$




\section{B. Computation of Local Reconstruction Filters}

Since we want to reconstruct the signal with the highest possible sampling, let $n_{R}=n_{\mathrm{CH}}$. We are focusing on a reconstruction approach that tries to recover the signal as described by reconstruction scheme $\mathbb{S}_{P}$ (17). In [11], where further reconstruction methods are also presented, it is referred to as "Projection Method."

When trying to solve the $n_{R} \times n_{S}$ matrix equation

$$
\mathbb{P H}=\mathbb{S}_{P}
$$

for the $n_{R} \cdot n_{\mathrm{CH}}$ reconstruction filters, we have to deal with its over-determination, since a pseudo inverse of $\mathbb{H}$ may not exist. To resolve this, we restrict ourselves to the subbands which will be recovered in the reconstructed spectrum. By this means we will properly reconstruct the major energy component of the signal, regardless of the impact of the reconstruction filters on the other spectral components. According to the reconstruction subbands $j \in\left\{n_{O}, \ldots, n_{O}+n_{R}-1\right\}$, we define the square matrix

$$
\widehat{\mathbb{H}}=\left(\begin{array}{ccc}
H_{0, n_{O}} & \cdots & H_{0, n_{O}+n_{R}-1} \\
\vdots & & \vdots \\
H_{n_{\mathrm{CH}}-1, n_{O}} & \ldots & H_{n_{\mathrm{CH}}-1, n_{O}+n_{R}-1}
\end{array}\right) .
$$

Looking at (17), we see that $\mathbb{P}$ should be the left inverse of $\mathbb{H}$. If $\operatorname{det} \widehat{\mathbb{H}} \neq 0$, it can be computed using the classical adjoint matrices of $\widehat{\mathbb{H}}$, and thus we obtain

$$
P_{k, i}(v, f)=(-1)^{(k+i)} \frac{m_{i, k}(\widehat{\mathbb{H}})}{\operatorname{det} \widehat{\mathbb{H}}}
$$

where $m_{i, k}(\widehat{\mathbb{H}})$ denotes the determinant of the matrix that arises from deleting row $i$ and column $k$ of $\widehat{\mathbb{H}}$.

In general, these $P_{k, i}$ fulfill (17) only for the subbands chosen above. The remaining entries of the reconstruction scheme (columns before $n_{O}$ and after $n_{O}+n_{R}-1$ ), which are likely to be nonzero, result in an undesired aliasing of the remaining subbands into the reconstruction bandwidth [as can be seen in (31)].

Note that this may result in energy being aliased from neighboring subbands (i.e., with respect to PRF), which may be folded onto the main lobe of the azimuth pattern, and thus cannot be dealt with using the standard bandpass filtering (as can be seen in Fig. 5). This kind of aliasing would not be present, had the signal been recorded with its sampling frequency after reconstruction, $n_{R} \cdot \mathrm{PRF}$. The choice of $B_{R}$ and the systems parameters (including PRF, azimuth pattern, and the number of channels) should be designed in such a way, that the energy of these contributions is negligible.

In order to compute the matrices $\widehat{\mathbb{H}}_{i, k}$ and the determinant of $\widehat{\mathbb{H}}$, we first determine the channel transfer functions by solving (3) for $H_{i}$, using the common abbreviation $\beta$ := $1-\left(f^{2} / v_{0}^{2} A\right)^{1 / 2}$ and Taylor approximation with respect to $v$ of the phase expression

$$
\begin{aligned}
H_{i}(\nu, f) \approx & E_{i}(\nu, f) E_{\text {ref }}^{-1}(\nu, f) \\
& \cdot \exp \left\{-2 \pi \mathrm{j} v_{0}\left(\tau_{0, \text { fit }, i} \beta_{i}-\tau_{0, \text { fit, ref }} \beta_{\text {ref }}\right)\right\} \\
& \cdot \exp \left\{-2 \pi \mathrm{j}\left(t_{0, \text { fit }, i}-t_{0, \text { fit, ref }}\right) f\right\}
\end{aligned}
$$

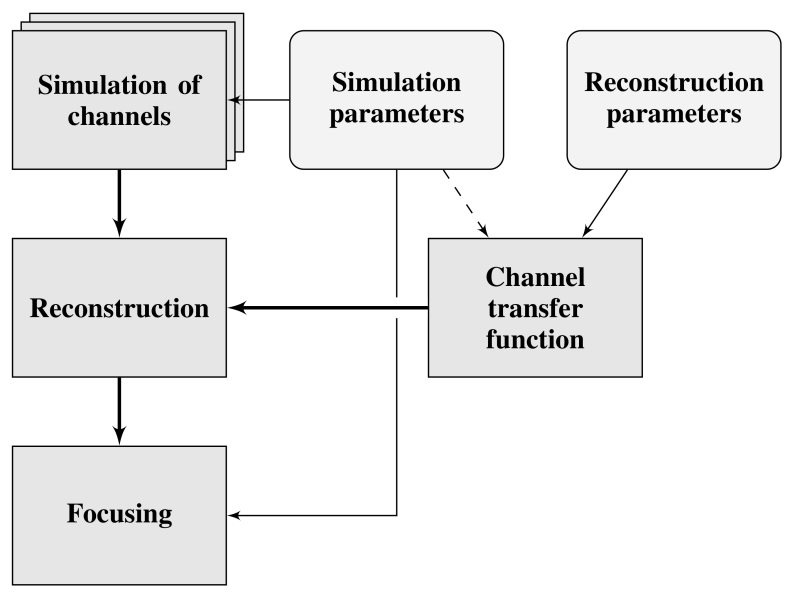

Fig. 1. Flow of the simulation framework.

$$
\begin{aligned}
& \cdot \exp \left\{-2 \pi \mathrm{j}\left(\frac{\tau_{0, \text { fit }, i}}{\beta_{i}}-\frac{\tau_{0, \text { fit, ref }}}{\beta_{\text {ref }}}\right) v\right\} \\
& \cdot \exp \left\{+\frac{\pi \mathrm{j} f^{2}}{\nu_{0}^{3}}\left(\frac{\tau_{0, \text { fit }, i}}{A_{i} \beta_{i}^{3}}-\frac{\tau_{0, \text { fit, ref }}}{A_{\text {ref }} \beta_{\text {ref }}^{3}}\right) v^{2}\right\} .
\end{aligned}
$$

Using this generic approach, the required reconstruction filters can be computed numerically.

Note that so far we have used the same set of parameters to compute both the aliased channel spectra $\mathcal{S}_{i}$ and the reconstruction filters $P_{k, i}$. In order to investigate the effect of the spatial variance of the reconstruction filters, we should use a set of simulation parameters to compute the former, and a different set of reconstruction parameters, corresponding to a reference target located at $\left(\tau_{0, \text { ref }}, t_{0, \text { ref }}\right)$, to compute the latter.

\section{Evaluation}

In order to assess the quality of the reconstruction filters, we focus the reconstructed signal $\mathcal{S}_{R}$. The focusing filter is computed using the full transfer function of the reference signal $\mathcal{S}_{I}$ according to the simulation parameters, as the deterioration observed in the resulting focused point spread function should reflect the quality of the recombination scheme only.

The resulting flow of the simulation framework is illustrated in Fig. 1. ${ }^{1}$

After the simulated scene is focused and transformed to the time domain, we can assess the quality of different reconstruction filters by means of a point target analysis of the focused result. Besides resolution (measured at $-3 \mathrm{~dB}$ ) and the peak to sidelobe ratio, in particular, the following quality parameters can be considered.

1) Signal's Phase at the Target's Position: For a perfectly reconstructed signal it should be zero (by design of the focusing filter), so we will refer to the measured value as "(peak) phase error."

2) Actual Peak Position: Similarly, the peak's position should be $(0 ; 0)$ and deviations indicate distortion of the impulse response.

\footnotetext{
${ }^{1}$ The dashed line will be explained in Section IV-A3.
} 
3) Azimuth Ambiguity to Signal Ratio (AASR): The signal's main energy is computed by integrating over a patch of size 32 by 32 pixels (compared to a geometrical resolution of 1.07). Since only the one target was simulated, all the remaining energy is considered an ambiguity. This allows us to compute the AASR.

\section{REsults}

In this section, we will compare different reconstruction results using the method under discussion. When computing the reconstruction filters $P_{k, i}$, we can compute $H_{i, j}$ up to the zeroth, first, or second order with respect to $v$, as shown in (24). We will refer to the resulting reconstruction filters computed using the simulation parameters by $P^{0}, P^{1}$, and $P^{2}$, respectively.

In addition, the use of the tilde symbol will indicate that the approximation $\beta \approx 1$ has been used (from now on referred to as " $\beta$-approximation"). This approximation is valid for a narrow azimuth bandwidth, i.e., $f \ll v_{0}$. If taken into account, as a function of $A$, it also contains information on the range migration, including its dependence on the targets range time [the linear term in (24)].

Be aware that the superscript does not correspond to the polynomial degree of the reconstruction filter: even the realization of a linear term in the channel transfer functions results in $P_{k, i}$ being a rational function with respect to $v$ with a denominator of degree $n_{R}$ and an enumerator of degree $n_{R}-1$.

Once the requirements for the application under investigation are determined, the generic equations (23) and (24) can be simplified, and implemented efficiently.

\section{A. Along Track Separation}

In the special TerraSAR-X mode referred to as DRA [9], the whole antenna is used to transmit, while at receive it is split, and two signals are recorded $\left(n_{\mathrm{CH}}=2\right)$. We can compute the channels' parameters making the following assumptions.

1) Both channels have the same pattern, so choose $E_{\text {ref }}(\nu, f)=E_{0}(\nu, f)=E_{1}(\nu, f)$.

2) $A_{\text {ref }}, \tau_{0, \text { fit,ref }}$, and $t_{0 \text {, fit,ref precisely describe the virtual }}$ reference signal as it would have been recorded by a single phase center antenna.

3) For receive phase center offsets $\pm \Delta x_{\text {sep }}$, we get the effective phase center offsets of $\pm\left(\Delta x_{\mathrm{sep}} / 2\right)$. Thus for a platform at velocity $v_{s}$ we can assume $A_{i}=A_{\text {ref }}$, $t_{0, \text { fit }, i}=t_{0, \text { fit,ref }} \pm\left(\Delta x_{\text {sep }} / 2 v_{s}\right)$.

We can approximate the increased closest range by

$$
\begin{aligned}
\tau_{0, \text { fit }, i} & =2 \sqrt{\left(\frac{\Delta x_{\text {sep }}}{2 c}\right)^{2}+\left(\frac{\tau_{0, \text { fit, ref }}}{2}\right)^{2}} \\
& =\tau_{0, \text { fit, ref }} \sqrt{\left(\frac{\Delta x_{\text {sep }}}{\tau_{0, \text { fit, ref } c}}\right)^{2}+1} \\
& \approx \tau_{0, \text { fit, ref }}\left(1+\frac{\Delta x_{\text {sep }}^{2}}{2 c^{2} \tau_{0, \text { fit,ref }}^{2}}\right) \\
& \approx \tau_{0, \text { fit, ref }}+\frac{\Delta x_{\text {sep }}^{2}}{2 c^{2} \tau_{0, \text { fit, ref }}} .
\end{aligned}
$$

TABLE II

TERRASAR-X DRA MODE

\begin{tabular}{|c|c|}
\hline Parameter & Value \\
\hline \hline$A_{\text {ref }}$ & $2.4250250675042497 \cdot 10^{-9}$ \\
\hline$\tau_{0, \text { fit,ref }}$ & $3.7359 \mathrm{~ms}$ \\
\hline$\Delta x_{\text {sep }}$ & $1.2 \mathrm{~m}$ \\
\hline PRF & $3000 \mathrm{~Hz}$ \\
\hline$f_{d c}$ & $0 \mathrm{~Hz}$ \\
\hline$\nu_{0}$ & $9.65 \mathrm{GHz}$ \\
\hline range bandwidth & $330 \mathrm{MHz}$ \\
\hline$\alpha_{\mathrm{U}}$ & 0.937 \\
\hline
\end{tabular}

Applying this to (24) yields

$$
\begin{aligned}
H_{i}(\nu, f) \approx \exp \left\{-2 \pi \mathrm{j} v_{0} \beta_{\text {ref }} \frac{\Delta x_{\text {sep }}^{2}}{2 c^{2} \tau_{0, \text { fit, ref }}} \mp 2 \pi \mathrm{j} \frac{\Delta x_{\text {sep }}}{2 v_{s}} f\right\} \\
\cdot \exp \left\{-2 \pi \mathrm{j} \frac{\Delta x_{\text {sep }}^{2}}{2 c^{2} \tau_{0, \text { fit, ref }} \beta_{\text {ref }}} v\right\} \\
\cdot \exp \left\{+\frac{\pi \mathrm{j} f^{2}}{v_{0}^{3}} \frac{\Delta x_{\text {sep }}^{2}}{2 c^{2} \tau_{0, \text { fit, ref }} A_{\text {ref }} \beta_{\text {ref }}^{3}} v^{2}\right\} .
\end{aligned}
$$

Using the $\beta$-approximation, and dropping the range frequency dependent terms altogether, we get an approximation equivalent to the one in $[8, \text { Sec. III-A, eq. (12) }]^{2}$

$$
\begin{array}{r}
\tilde{H}_{i}(\nu, f) \approx \exp \left\{-\pi \mathrm{j} v_{0} \frac{\Delta x_{\text {sep }}^{2}}{c^{2} \tau_{0, \text { fit, ref }}}\right\} \\
\cdot \exp \left\{\mp \pi \mathrm{j} \frac{\Delta x_{\text {sep }}}{v_{s}} f\right\} .
\end{array}
$$

This approximation is valid for small $\Delta x_{\text {sep }}$, small $f$ and high carrier frequency $v_{0}$. According to the naming convention introduced in the first paragraph of this section, the reconstruction filter based on this approximation will be denoted by $\tilde{P}^{0}$.

Note that for $\beta \approx 1$ the dropped linear term corresponds to a shift in range induced by the signals increased round trip time at the time of the closest approach, due to the phase center separation [see (25)]. Since it is the same for both channels, it can be accounted for in the final registration of the focused result.

In the following, two simulation cases with different squint modes will be presented.

1) Zero Squint: We choose simulation parameters matched to the acquisition geometry of the TerraSAR-X DRA mode (see Table II).

We will be simulating two channels, each on eight subbands centered around the Doppler-centroid, so we have $n_{C H}=2$ and $n_{S}=8$. Since we want to properly reconstruct the signals main energy component (i.e., the fourth and fifth subband; as can be seen in Fig. 5) we choose $n_{O}=3$. In order to highlight the effects of the additional reconstruction aliasing, we set the antenna pattern's peak-to-zero width to 2 . PRF. For better comparison, this pattern is kept fixed throughout our analysis.

\footnotetext{
${ }^{2}$ For comparison note that $\left(1 / \tau_{0, \text { fit, ref })} \approx\left(c / 2 R_{0}\right)\right.$ and $\left(v_{0} / c\right)=(1 / \lambda)$.
} 
TABLE III

Point TARget ANALYSIS For Zero SQUint

\begin{tabular}{|l|r|r|}
\hline Parameter & $\tilde{P}^{0}$ & $P^{2}$ \\
\hline \hline peak phase error [deg] & $-1.942 \cdot 10^{-4}$ & $-1.942 \cdot 10^{-4}$ \\
\hline rg peak position [px] & $1.015 \cdot 10^{-5}$ & $6.929 \cdot 10^{-6}$ \\
\hline az peak position [px] & $1.170 \cdot 10^{-6}$ & $1.170 \cdot 10^{-6}$ \\
\hline $\begin{array}{l}\text { rg peak width [px] } \\
\text { (ideal: } 1.07442)\end{array}$ & 1.07441 & 1.07441 \\
\hline $\begin{array}{l}\text { az peak width [px] } \\
\text { (ideal: } 1.07388 \text { ) }\end{array}$ & 1.07386 & 1.07386 \\
\hline $\begin{array}{l}\text { rg PSLR [dB] } \\
\text { (ideal: }-18.6438)\end{array}$ & -18.6437 & -18.6437 \\
\hline $\begin{array}{l}\text { az PSLR [dB] } \\
\text { (ideal: }-18.6476)\end{array}$ & -18.6486 & -18.6486 \\
\hline
\end{tabular}

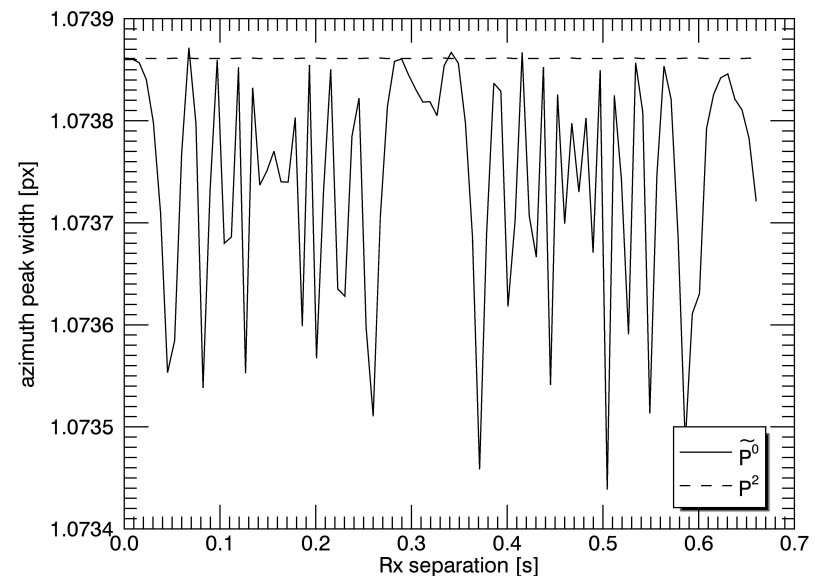

Fig. 2. Azimuth resolution as a function of the receive antenna separation time.

Table III compares the results of the point target analysis corresponding to the reconstruction filters $\tilde{P}^{0}$ and $P^{2}$. The values given in parentheses are the ideal values computed by measuring the Fourier-transformation of the used Hammingwindow. We see no significant deterioration of the focusing quality.

Future multistatic SAR systems may consist of multiple satellites traveling on the same orbit, increasing the value of $\Delta x_{\text {sep }}$ significantly. We ran simulations with an along track separation time of up to $0.7 \mathrm{~s}$. While the reconstruction with $\tilde{P}^{0}$ exhibits very small numerical variations in the measured parameters, we still cannot observe any significant loss of focusing quality compared to $P^{2}$. Fig. 2 shows the azimuth resolution, as an example. Note that the variation is less than half a millipixel.

However, due to an increased contribution of the additional reconstruction aliasing as described in (20), we can observe a variation of the AASR (Fig. 3).

We now introduce a measure of the effective sampling uniformity

$$
\begin{aligned}
\alpha_{\mathrm{U}} & :=2 \cdot\left(\frac{\left|t_{0, \mathrm{fit}, 0}-t_{0, \mathrm{fit}, 1}\right|}{\mathrm{PRI}} \bmod 1\right) \\
& =2 \cdot\left(\frac{\left|\Delta x_{\mathrm{sep}}\right|}{v_{s} \text { PRI }} \bmod 1\right)
\end{aligned}
$$

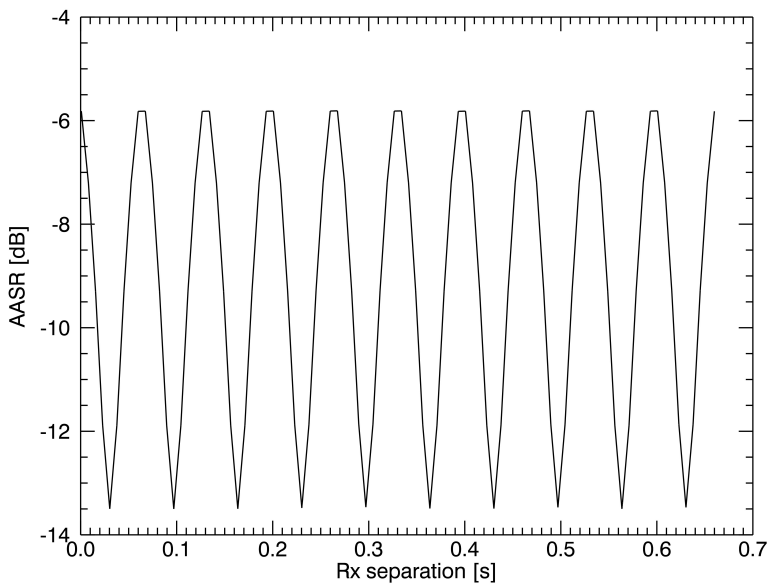

Fig. 3. AASR as a function of the receive antenna separation time.

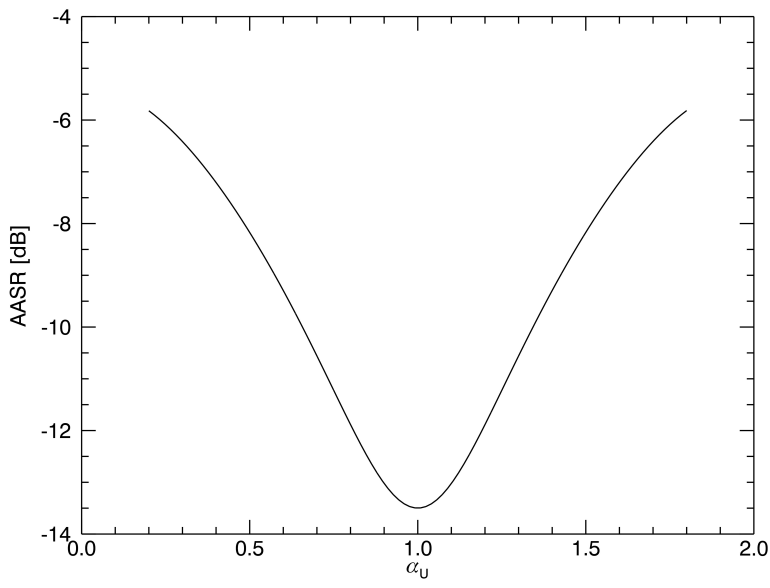

Fig. 4. AASR as a function of the effective sampling uniformity.

where $\alpha_{\mathrm{U}}=0$ indicates coinciding sample positions and $\alpha_{\mathrm{U}}=1$ uniform sampling. Note that the parameter $t_{0, \text { fit, }}$. in general depends on the targets position, hence "effective" (as in "effective platform velocity").

Reorganizing the simulated results, we can identify $\alpha_{U}$ as being the determining factor for a good AASR (Fig. 4).

This is no surprise, as the amplitude of det $\widehat{\mathbb{H}}$ (after factoring out all terms with amplitude 1) is given by

$$
\begin{aligned}
|\operatorname{det} \widehat{\mathbb{H}}| & =\left|H_{0, n_{O}} H_{1, n_{O}+1}-H_{0, n_{O}+1} H_{1, n_{O}}\right| \\
& =\left|\exp \left\{\pi \mathrm{j} \omega_{U}\right\}-\exp \left\{-\pi \mathrm{j} \omega_{U}\right\}\right| \\
& =2\left|\sin \left(\pi \omega_{U}\right)\right| \\
& =2 \sin \left(\pi\left(\left|\omega_{U}\right| \bmod 1\right)\right) \\
& =2 \sin \left(\pi \frac{\alpha_{U}}{2}\right)
\end{aligned}
$$

where

$$
\begin{aligned}
\omega_{U} & =\frac{\Delta x_{\mathrm{sep}}}{v_{s}}\left(f+n_{O} \mathrm{PRF}\right)-\frac{\Delta x_{\mathrm{sep}}}{v_{s}}\left(f+\left(n_{O}+1\right) \mathrm{PRF}\right) \\
& =-\frac{\Delta x_{\mathrm{sep}}}{v_{s}} \mathrm{PRF}=-\frac{\Delta x_{\mathrm{sep}}}{v_{s} \mathrm{PRI}} .
\end{aligned}
$$

This means that if the effective sampling uniformity is zero, then so is $\operatorname{det} \widehat{\mathbb{H}}$, and our set of reconstruction equations (17) has no solution. 


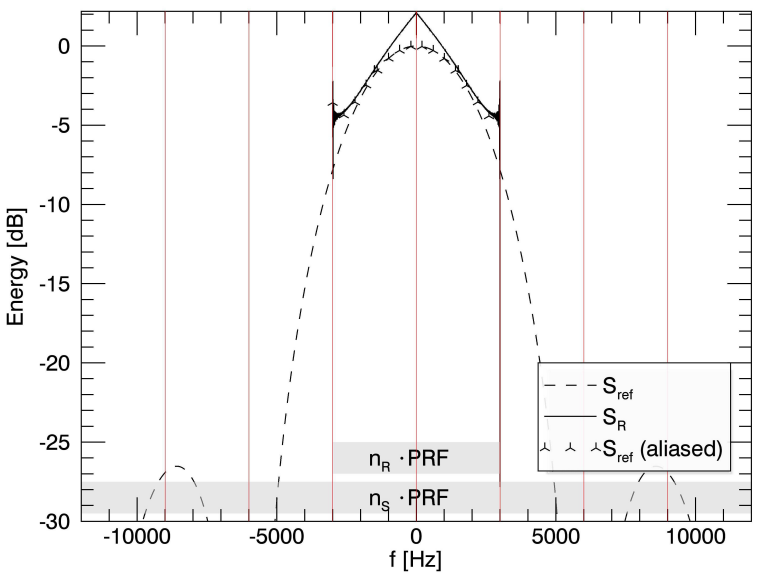

Fig. 5. Azimuth profile for $\alpha_{U}=0.2$.

As we increase $\Delta x_{\text {sep }}$ to $2.81 \mathrm{~m}$ while keeping the remaining parameters fixed (thus having $\alpha_{\mathrm{U}} \approx 0.2$ ), $|\operatorname{det} \widehat{\mathbb{H}}|$ decreases, which in turn results in a larger magnitude of the reconstruction filters. By definition of the reconstruction filters, the signal component will be unchanged; the aliased component, however, will be amplified, thus increasing the AASR. To illustrate this, we take a look at the average energy of the corresponding reconstruction scheme

$$
E[\mathbb{S}] \approx\left(\begin{array}{lllllllll}
9.5 & 6.9 & 3.6 & 1.0 & 0.0 & 1.0 & 3.6 & 6.9 \\
6.9 & 3.6 & 1.0 & 0.0 & 1.0 & 3.6 & 6.9 & 9.5
\end{array}\right)
$$

While the center coefficients forming a 2 by 2 identity matrix reflect the proper reconstruction of the signal component, the increased off-center entries correspond to a strong additional aliasing according to (20).

Looking at the azimuth profile of the reconstructed signal (Fig. 5), we can see the energy resulting from this unfavorable combination of $\Delta x_{\text {sep }}$ and the PRF, especially at the center frequency.

Note that - by choice of the pattern width - this aliased energy does not result from an aliasing with respect to $n_{R}$ PRF. For a much larger phase center separation of $\Delta x_{\text {sep }}=$ $116.57 \mathrm{~m}$, which is corresponding to $\alpha_{\mathrm{U}} \approx 1.0$, we get a reconstruction scheme which does not introduce additional aliased energy

$$
E[\mathbb{S}] \approx\left(\begin{array}{lllllllll}
0.0 & 1.0 & 0.0 & 1.0 & 0.0 & 1.0 & 0.0 & 1.0 \\
1.0 & 0.0 & 1.0 & 0.0 & 1.0 & 0.0 & 1.0 & 0.0
\end{array}\right) \text {. }
$$

2) Squinted DRA: Let us consider the possibility of a squinted DRA acquisition by changing the Doppler-centroid to $3 \mathrm{kHz}$. Due to the higher azimuth frequencies, we can measure the effects of the approximations made in (27).

Focusing the reconstruction results according to $\tilde{P^{0}}, P^{0}$, $P^{1}$, and $P^{2}$, we can observe small phase errors at the signal's peak, increasing with the separation time (Fig. 6). The main error disappears once we move from $\tilde{P}^{0}$ to $P^{0}$ (dropping the assumption $\beta(f) \approx 1$ ). The remaining jitter is removed by considering the linear term in (24), after which the measured phase is below $10^{-4}$ degree.

Since the $\beta$-approximation is assuming $f<<v_{0}$, we repeat the simulation with parameters matching an L-band SAR

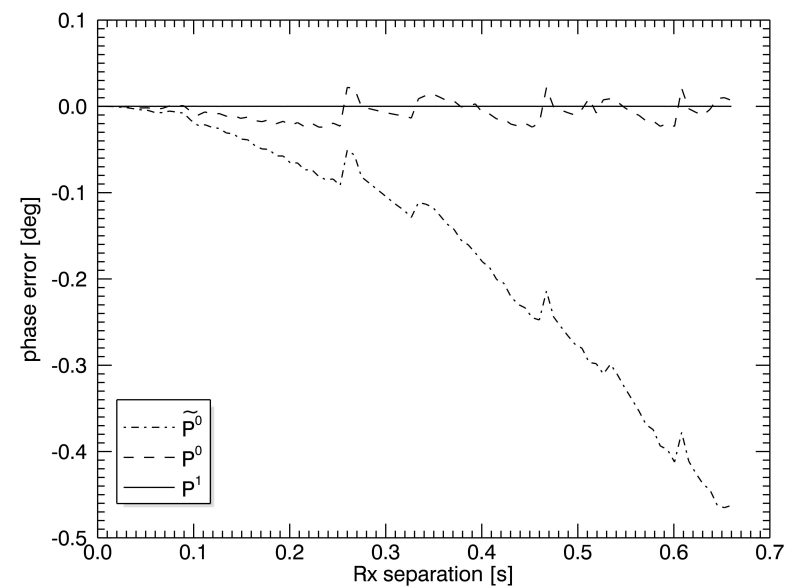

Fig. 6. Peak phase error for squinted acquisition.

TABLE IV

L-BAND PARAMETERS

\begin{tabular}{|c|c|}
\hline Parameter & Value \\
\hline \hline$A_{\text {ref }}$ & $2.4250 \cdot 10^{-9}$ \\
\hline$\tau_{0, \text { fit,ref }}$ & $3.7359 \mathrm{~ms}$ \\
\hline$\Delta x_{\text {sep }}$ & $3 \mathrm{~m}-2536 \mathrm{~m}$ \\
\hline $\mathrm{PRF}$ & $3000 \mathrm{~Hz}$ \\
\hline$f_{d c}$ & $400 \mathrm{~Hz}$ \\
\hline$\nu_{0}$ & $1.25 \mathrm{GHz}$ \\
\hline range bandwidth & $80 \mathrm{MHz}$ \\
\hline
\end{tabular}

TABLE V

L-BAND RESULTS

\begin{tabular}{|c|c|}
\hline Reconstruction & Max. Phase Error \\
\hline \hline$\tilde{P}^{0}$ & $1.023^{\circ}$ \\
\hline$P^{0}$ & $0.035^{\circ}$ \\
\hline$P^{1}$ & $0.018^{\circ}$ \\
\hline$P^{2}$ & $0.018^{\circ}$ \\
\hline
\end{tabular}

with the same orbital and comparable acquisition parameters (see Table IV). Decreasing $\nu_{0}$ from 9.65 to $1.25 \mathrm{GHz}$ increases the impact of the $\beta$-approximation. We see the exact same behavior with regards to AASR and resolution as in $\mathrm{X}$-band (i.e., the AASR is determined by $\alpha_{\mathrm{U}}$ and no significant peak widening is observed). Analyzing the peak phase we get a qualitatively similar result to that shown in Fig. 6, with slightly increased maximal absolute phase errors listed in Table V.

3) Spatial Variation: When computing the reconstruction filters, we are faced with a problem very common in SAR processing. The signals are being described in spectral domain, yet their parameters are spatially varying.

In the $D R A$-like case described in (26), the reconstruction filters $H_{i}$ depend on the parameters $A_{\text {ref }}$ and $\tau_{0, \text { fit,ref. The }}$ former corresponds to a great extent to the effective platform velocity, and thus must be updated along range; the latter describes the signals minimal round trip time, which obviously is also range dependent.

Range-Doppler domain-based SAR processing algorithms can be classified by whether they compensate the Taylor terms 
of the point response function range dependently, at a reference point, or not at all.

The most basic Range-Doppler Algorithm realizes a range dependent azimuth focusing (the constant Taylor term), corrects the range migration at some reference point (the linear Taylor term), and ignores higher order terms. When Chirpscaling is applied, the range migration is corrected range dependently, and a reference secondary range compression is applied. In modern high resolution SAR processors all the Taylor terms including the secondary range compression can be corrected range dependently using a cubic scaling technique [12] and a residual correction is applied for all higher order terms at a reference point [13].

As mentioned in the last paragraph of Section III-B, we are maintaining two sets of parameters: the simulation parameters and the reconstruction parameters. Depending on which of those we use to compute the different terms of (24), we can simulate a range dependent or referenced realization of the respective term when the reconstruction filters are computed. The use of the simulation parameters corresponds to the range dependent realization and is indicated by the dashed line in Fig. 1.

Extending the notation introduced at the beginning of this chapter, $P^{n . m}$ will denote the reconstruction filters based on the first $n$ terms of $H_{i, j}$ computed range dependently, and the remaining terms up to the $m$ th for a reference range. We will write "NO" to indicate that no corresponding terms were computed at all.

Let us illustrate this for some filters of special relevance. The cheapest possible processing [as shown in (27)] is denoted by $\tilde{P}^{N O .0}$. Using the $\beta$-approximation, no range frequency dependent terms and the constant term is computed at the reference position. We will later (Section IV-B2) compare processing results according to $P^{0.1}$ and $P^{1 . N O}$. For both of these, (24) is computed up to the linear term. For $P^{0.1}$, the constant term is computed range dependently (i.e., using the simulation parameters) and the linear term is computed for the reference range (using the reconstruction parameters). For $P^{1 . N O}$ both terms are computed using the simulation parameters and no higher terms are considered.

Keeping the reconstruction parameters fixed, while changing the simulation parameters according to a swath of width $100 \mathrm{~km}$, using the parameters from Table II, we do not see any deterioration even at the swath edges when the processing filters $\tilde{P}^{\mathrm{NO} .0}$ are used. This can be expected, since differentiating the only range dependent term in (27) shows that it changes for about $2^{\circ} / \mathrm{s}$ trip time, i.e., $1.3 \cdot 10^{-8} / \mathrm{m}$ slant range (at the reference range).

However, since this increases quadratically with the phase center offset $\Delta x_{\text {sep }}$, we next simulate data according to an along track baseline of $2 \cdot 1141.36 \mathrm{~m}$ (chosen to facilitate an effective sampling uniformity of $\left.\alpha_{U}=0.999\right)$ and reconstruct it using all possible reconstruction filters.

Investigating the simulation results, we see that we must at least realize the constant term in a range dependent manner, as the phase error is now more than $\pm 200^{\circ}$ at the swath edges (according to a phase rate of about $1.2 \cdot 10^{-2} / \mathrm{m}$ slant range). Introducing the range dependent realization of the

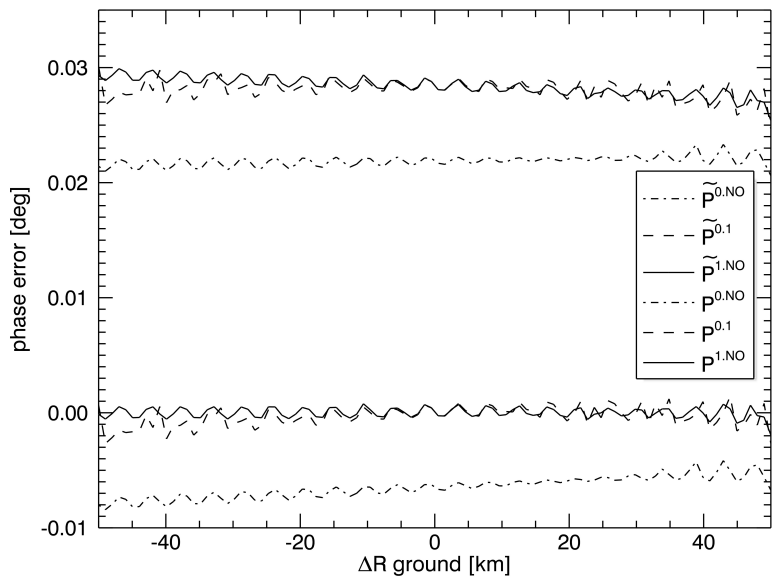

Fig. 7. Different phase errors over range offset. The top three legend entries refer to the top three plots incorporating the $\beta$-approximation.

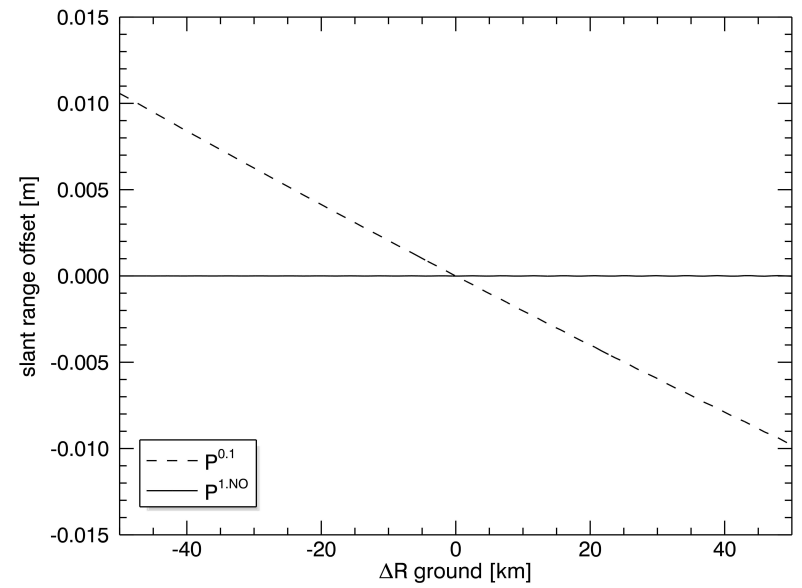

Fig. 8. Slant range error over range offset.

constant term, the phase error is below $0.03^{\circ}$. On the other hand, we can measure no effect of the second order term of (24), leaving us with six remaining reconstruction options. Their respective phase errors are shown in Fig. 7.

Comparing the results produced by filters adopting the $\beta$-approximation (in the upper part of Fig. 7) to their respective counterparts, we conclude that using $\beta=1$ in the constant term of (24) introduces a shift of about $0.03^{\circ}$ (as it appears in all three of them). Similarly, we see that in either case the omission of the linear term introduces an even smaller offset of $-0.01^{\circ}$ (which happens to compensate part of the larger $\beta$-approximation error in this case). The phase error appears unbiased and well below $0.005^{\circ}$, once the linear term is incorporated. We cannot see a significant improvement by updating it with range.

Updating the linear term with range has a small effect on the range position of the focused result, as a linear range error of up to $0.01 \mathrm{~m}$ is removed (Fig. 8). Of course, this error could be compensated by adjusting the range pixel spacing of the reconstructed signal.

\section{B. Cross Track Separation}

In this section, we will investigate the recombination of two channels simulated using parameters according to two 
TABLE VI

TANDEM-X

\begin{tabular}{|c|c|c|}
\hline Parameter & Value master & Value slave \\
\hline \hline$A$ & $2.4066328 \cdot 10^{-9}$ & $2.4065071 \cdot 10^{-9}$ \\
\hline$\tau_{0, \text { fit }}$ & $4.8089 \mathrm{~ms}$ & $4.8097 \mathrm{~ms}$ \\
\hline$t_{0, \text { fit }}$ & $16.011166 \mathrm{~ms}$ & $7.6017415 \mathrm{~ms}-$ \\
& & $7.9350021 \mathrm{~ms}$ \\
\hline
\end{tabular}

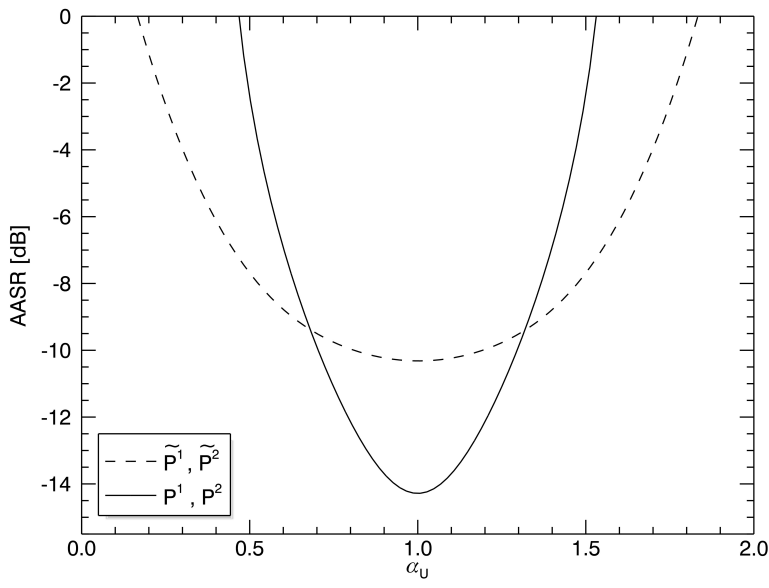

Fig. 9. AASR as function of the effective sampling uniformity.

orbits of the TanDEM-X mission with a perpendicular baseline of $76.6 \mathrm{~m}$ (see Table VI).

In this scenario one satellite is transmitting and both are receiving. The channel received by the transmitting satellite is referred to as "master," the other one as "slave." The master channel is also used as the reference signal.

1) Along Track Displacement: In order to investigate the effect of the along-track displacement, the slave satellites position vectors are shifted in time by up to ( $2 / \mathrm{PRF})$. Since the two channels have different closest approach round trip times $\tau_{0, \text { fit }}$, processing with $P^{0}$ is futile. Plotting the AASR as a function of the effective sampling uniformity for the remaining processing variants (Fig. 9), we can make three observations.

1) Although the interleaved phase centers of the recorded channels do not correspond to one uniformly sampled signal (due to the different orbits), the parameter $\alpha_{U}$ still governs the reconstruction quality in terms of AASR, and a meaningful reconstruction is only possible for a narrower range of $\alpha_{\mathrm{U}}$.

2) The $\beta$-approximation $(\tilde{P})$ worsens the optimum AASR by $4 \mathrm{~dB}$, but causes less deterioration for suboptimal effective sampling uniformity. This reduction of the additional reconstruction-induced aliasing energy comes at the cost of signal reconstruction accuracy [(17) is no longer fulfilled], in this case leading to a peak phase error of at least $7.9^{\circ}$.

3) The realization of the quadratic term has no measurable effect.

2) Spatial Variation: We now choose the along track separation according to an effective sampling uniformity of one, and vary the simulation parameters in the same way as in Section IV-A3. Keeping in mind the previous results, we only

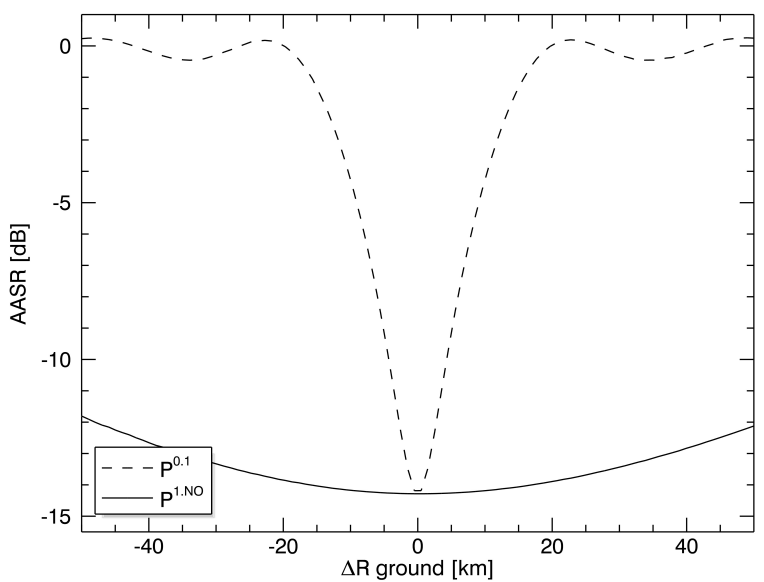

Fig. 10. AASR as function of the range offset.

TABLE VII

RECONSTRUCTION COMPLEXITY

\begin{tabular}{|l|l|l|}
\hline Term & zero Baseline [IV-A] & $76.6 \mathrm{~m}$ Baseline [IV-B] \\
\hline \hline $\begin{array}{l}\text { exact } \beta \\
(\tilde{P} \rightarrow P)\end{array}$ & necessary & necessary \\
\hline $\begin{array}{l}\text { fonear squinted acquisitions } \\
\left(P^{0} \rightarrow P^{1}\right)\end{array}$ & $\begin{array}{l}\text { marginal improvement } \\
\text { for squinted acquisitions if } \\
\text { realized at reference range }\end{array}$ & $\begin{array}{l}\text { necessary } \\
\text { update with range }\end{array}$ \\
\hline
\end{tabular}

have to investigate the processing filters $P^{0.1}$ and $P^{1 . N O}$, i.e., using a constant linear term versus a range dependent linear term, and ignoring higher orders (as explained in Section IV-A3).

Looking at Fig. 10, we can see that a constant linear range term has a dramatic effect on the AASR. A reasonable swath width cannot be processed without accounting for it is spatial variation. If we do use $P^{1 . N O}$-processing, we increase the performance significantly. We only observe a small variation of the AASR.

To understand this variation of the AASR, we need to examine the behavior of the parameters $t_{0, \text { fit }, i}$. These parameters represent the times when the shortest delay is observed by the sensor [recall (1)]. Due to the sensor's motion during the pulse propagation, they not only vary with range, but the rate of their change depends on the acquisition geometry [14]. In the case under consideration, the difference of the zero-Doppler times $t_{0, \text { fit, } 0}$ and $t_{0, \text { fit, } 1}$ decreases by about $70 \mu$ s from near range to far range. As the effective sampling uniformity is derived from this difference [see (28)], it varies from 0.8 to 1.2. The observed AASR variation is consistent with these values of $\alpha_{U}$ (compare Fig. 9).

\section{CONClusion}

A spectral domain simulation scheme has been proposed, that can help to assess the quality of various reconstruction filters. It assumes that the SAR signals echo delay time can be described using hyperbolic functions, and thus gives an upper boundary on the reconstruction performance. Case studies have been presented using parameters corresponding to acquisition geometries inspired by current TerraSAR-X and TanDEM-X imaging modes, and requirements and limitations for appropriate reconstruction filters were derived. 
The introduced effective sampling uniformity $\alpha_{\mathrm{U}}$ has been shown to be a determining factor for reconstruction quality, especially for configurations with nonzero perpendicular baseline. For appropriate values of $\alpha_{\mathrm{U}}$, the qualitative effects of the $\beta$-approximation and the realization of the different Taylor terms of the channel transfer functions on the reconstruction quality have been investigated. The effects of the $\beta$-approximation and the linear Taylor term are summarized in Table VII, while the consideration of the second order Taylor term had no effect in any of the investigated cases.

\section{REFERENCES}

[1] I. G. Cumming and F. H. Wong, Digital Processing of Synthetic Aperture Radar Data: Algorithms and Implementation. Norwood, MA, USA: Artech House, 2005.

[2] A. Meta, J. Mittermayer, P. Prats, R. Scheiber, and U. Steinbrecher, "TOPS imaging with TerraSAR-X: Mode design and performance analysis," IEEE Trans. Geosci. Remote Sens., vol. 48, no. 2, pp. 759-768, Feb. 2010.

[3] D. Geudtner, R. Torres, P. Snoeij, M. Davidson, and B. Rommen, "Sentinel-1 system capabilities and applications," in Proc. IEEE Int. Geosci. Remote Sens. Symp. (IGARSS), Quebec City, QC, Canada, Jul. 2014, pp. 1457-1460.

[4] M. Villano, G. Krieger, and A. Moreira, "Staggered SAR: High-resolution wide-swath imaging by continuous PRI variation," IEEE Trans. Geosci. Remote Sens., vol. 52, no. 7, pp. 4462-4479, Jul. 2014

[5] N. Gebert and G. Krieger, "Ultra-wide swath SAR imaging with continuous PRF variation," in Proc. IEEE 8th Eur. Conf. Synth. Aperture Radar (EUSAR), Aachen, Germany, Jun. 2010, pp. 966-969.

[6] J. Brown, "Multi-channel sampling of low-pass signals," IEEE Trans. Circuits Syst., vol. 28, no. 2, pp. 101-106, Feb. 1981.

[7] G. Krieger, N. Gebert, and A. Moreira, "Unambiguous SAR signal reconstruction from nonuniform displaced phase center sampling," IEEE Geosci. Remote Sens. Lett., vol. 1, no. 4, pp. 260-264, Oct. 2004.

[8] N. Gebert, G. Krieger, and A. Moreira, "Digital beamforming on receive: Techniques and optimization strategies for high-resolution wide-swath SAR imaging," IEEE Trans. Aerosp. Electron. Syst., vol. 45, no. 2, pp. 564-592, Apr. 2009.

[9] J. H. Kim, M. Younis, P. Prats-Iraola, M. Gabele, and G. Krieger, "First spaceborne demonstration of digital beamforming for azimuth ambiguity suppression," IEEE Trans. Geosci. Remote Sens., vol. 51, no. 1, pp. 579-590, Jan. 2013.

[10] F. He, Q. Chen, Z. Dong, and Z. Sun, "Processing of ultrahigh-resolution spaceborne sliding spotlight SAR data on curved orbit," IEEE Trans. Aerosp. Electron. Syst., vol. 49, no. 2, pp. 819-839, Apr. 2013.
[11] D. Cerutti-Maori, I. Sikaneta, J. Klare, and C. H. Gierull, "MIMO SAR processing for multichannel high-resolution wide-swath radars," IEEE Trans. Geosci. Remote Sens., vol. 52, no. 8, pp. 5034-5055, Aug. 2014.

[12] G. W. Davidson, I. G. Cumming, and M. R. Ito, "A chirp scaling approach for processing squint mode SAR data," IEEE Trans. Aerosp. Electron. Syst., vol. 32, no. 1, pp. 121-133, Jan. 1996.

[13] H. Breit, M. Fischer, U. Balss, and T. Fritz, "TerraSAR-X staring spotlight processing and products," in Proc. IEEE 10th Eur. Conf. Synth. Aperture Radar (EUSAR), Berlin, Germany, Jun. 2014, pp. 1-4.

[14] H. Breit, T. Fritz, U. Balss, M. Lachaise, A. Niedermeier, and M. Vonavka, "TerraSAR-X SAR processing and products," IEEE Trans. Geosci. Remote Sens., vol. 48, no. 2, pp. 727-740, Feb. 2010.

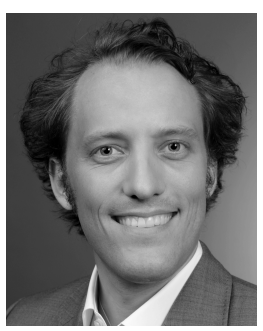

Moritz Kiemer received the Diploma degree in mathematics from the Ludwig-MaximiliansUniversity of Munich, Munich, Germany, in 2010.

Since 2011, he has been a Research Assistant with the Department of Photogrammetry and Remote Sensing, Technische Universität München, Munich. He is currently developing synthetic aperture radar (SAR) focusing algorithms for the TanDEM-X mission and future SAR satellites at the German Aerospace Center, Weßling, Germany.

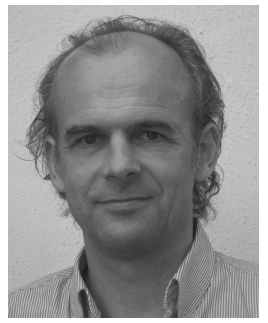

Helko Breit received the Diploma degree in electrical and telecommunication science from the Technical University of Munich, Munich, Germany, in 1990.

Since 1990, he has been with the German Aerospace Center, Weßling, Germany, where he is currently supervising the Synthetic Aperture Radar (SAR) Processor Development Team at the Remote Sensing Technology Institute. He was involved in a variety of international missions, including SIR-C/X-SAR, SRTM/X-SAR, and the German missions TerraSAR-X/TanDEM-X. He contributed in several European Space Agency studies and supported the commissioning of the ESA's Sentinel 1 SAR satellites. He was responsible for the development of the TerraSAR-X multimode SAR processor TMSP and the bistatic processing of TanDEM-X mission data. His research interests include the development of SAR processing algorithms and systems for the future SAR satellites and missions such as HRWS and Tandem-L. 